\title{
Transcriptional development of phospholipid and lipoprotein metabolism in different intestinal regions of Atlantic salmon (Salmo salar) fry
}

Yang Jin ${ }^{1 *}$ (D), Rolf Erik Olsen ${ }^{1}$, Mari-Ann Østensen ${ }^{1}$, Gareth Benjamin Gillard ${ }^{3}$, Sven Arild Korsvoll ${ }^{4}$, Nina Santi ${ }^{4}$, Arne Bjørke Gjuvsland ${ }^{2}$, Jon Olav Vik², Jacob Seilø Torgersen ${ }^{4}$, Simen Rød Sandve ${ }^{2}$ and Yngvar Olsen ${ }^{1}$

\begin{abstract}
Background: It has been suggested that the high phospholipid (PL) requirement in Atlantic salmon (Salmo salar) fry is due to insufficient intestinal de-novo synthesis causing low lipoprotein (LP) production and reduced transport capacity of dietary lipids. However, in-depth ontogenetic analysis of intestinal PL and LP synthesis with the development of salmon has yet to be performed. Therefore, in this paper we used RNA-Seq technology to investigate the expression of genes involved in PL synthesis and LP formation throughout early developmental stages and associate insufficient expression of synthesis pathways in salmon fry with its higher dietary PL requirement. There was a special focus on the understanding homologous genes, especially those from salmonid-specific fourth vertebrate whole-genome duplication (Ss4R), and their contribution to salmonid specific features of regulation of PL metabolic pathways. Salmon fry were sampled at $0.16 \mathrm{~g}$ ( 1 day before first-feeding), 2.5 and $10 \mathrm{~g}$ stages of development and transcriptomic analysis was applied separately on stomach, pyloric caeca and hindgut of the fish.
\end{abstract}

Results: In general, we found up-regulated pathways involved in synthesis of phosphatidylcholine (PtdCho), phosphatidylethanolamine (PtdEtn), and LP in pyloric caeca of salmon between 0.16 and $10 \mathrm{~g}$. Thirteen differentially expressed genes $(q<0.05)$ in these pathways were highly up-regulated in $2.5 \mathrm{~g}$ salmon compared to $0.16 \mathrm{~g}$, while only five more differentially expressed $(q<0.05)$ genes were found when the fish grew up to $10 \mathrm{~g}$. Different homologous genes were found dominating in stomach, pyloric caeca and hindgut. However, the expression of dominating genes in pathways of PL and LP synthesis were much higher in pyloric caeca than stomach and hindgut. Salmon-specific homologous genes (Ss4R) had similar expression during development, while other homologs had more diverged expression.

Conclusions: The up-regulation of the de-novo PtdCho and PtdEtn pathways confirm that salmon have decreasing requirement for dietary PL as the fish develops. The similar expressions between Ss4R homologous genes suggest that the functional divergence of these genes was incomplete compared to homologs derived from other genome duplication. The results of the present study have provided new information on the molecular mechanisms of phospholipid synthesis and lipoprotein formation in fish.

Keywords: Atlantic salmon, Biosynthesis, Gene expression, Homologous genes, Intestinal regions, Lipoprotein, Phospholipids, RNA-Seq, Whole genome duplication

\footnotetext{
* Correspondence: yang.jin@ntnu.no

${ }^{1}$ Department of Biology, Faculty of Natural Sciences, Norwegian University of

Science and Technology, NO-7491 Trondheim, Norway

Full list of author information is available at the end of the article
}

(c) The Author(s). 2018 Open Access This article is distributed under the terms of the Creative Commons Attribution 4.0 International License (http://creativecommons.org/licenses/by/4.0/), which permits unrestricted use, distribution, and reproduction in any medium, provided you give appropriate credit to the original author(s) and the source, provide a link to the Creative Commons license, and indicate if changes were made. The Creative Commons Public Domain Dedication waiver (http://creativecommons.org/publicdomain/zero/1.0/) applies to the data made available in this article, unless otherwise stated. 


\section{Background}

Phospholipids (PL) are the main constituent of all biological cell membranes and separate the intracellular and extracellular aqueous environments. In addition to providing a structural scaffold for cell membranes, PL are also involved in numerous biological functions, such as provision of metabolic energy, cell membrane transport, and regulation of metabolism [1]. PL are also key structural components of lipoproteins (LP), which are involved in transport of dietary lipids from intestinal enterocytes to liver and peripheral tissues [2, 3]. It is well known that dietary inclusions of PL can improve growth and performance in many fish species including Atlantic salmon (Salmo salar) [4], Atlantic cod (Gadus morhua) [5], and rainbow trout (Oncorhynchus mykiss) [6]. Dietary and biliary PL is mainly digested by intestinal phospholipase $\mathrm{A}_{2}$ in fish, resulting in 1-acyl lyso-phospholipids (lysoPL) and free fatty acids (FFA). Subsequently, both lyso-PL and FFA are absorbed into intestinal enterocytes and reesterified into PL before being exported to the rest of the body [7]. In addition, PL can be synthesized de-novo in enterocytes from glycerol-3-phosphate (G-3-P) in fish [8].

A minimum requirement of $\mathrm{PL}$ is associated with early developmental stages of fish, but so far no minimum requirement has been demonstrated in adult fish [8]. In line with this, dietary PL has been shown to enhance growth and survival in Atlantic salmon fry up to $2.5 \mathrm{~g}$, but not in larger fish [4, 9]. It has been suggested that the higher PL requirement is due to insufficient ability of de-novo synthesis in the intestine leading to low LP production and consequently reduced transport capacity of dietary lipids [8]. This was supported by previous histological studies in salmonids, showing lipid accumulation in intestinal enterocytes when fed PL deficient diet $[9,10]$. However, these differences were not evident in a previous transcriptomic study where gene expressions in PL biosynthesis pathways were unchanged in $2.5 \mathrm{~g}$ salmon fed by PL-supplemented diet [11]. No study has examined the gene expression in salmon smaller than $2.5 \mathrm{~g}$.

The intestinal tract of salmon consists of several regions, with different functions in lipid digestion, absorption and transport. It is generally believed that pyloric caeca (PC), rather than stomach (SM) or hindgut (HG), is the predominant region for lipid absorption and transport in salmon $[12,13]$. Therefore, PL and LP were assumed to be mostly synthesized in $\mathrm{PC}$ region. However, other tissues like SM and HG could also have ability of synthesizing PL due to its structural roles in cell membranes [1]. LP has occasionally been observed in hindgut, suggesting some lipid absorption and transport activities in the region [14]. So far no study has demonstrated the expression of PL metabolic pathways in SM and HG of fish.
Many homologous genes in mammals were found belong to gene families controlling the same enzymatic processes but have distinct regulation in different tissues and developmental stages [1]. In this respect the Atlantic salmon has another layer of functional genome complexity as it experienced two extra rounds of whole genome duplication (WGD) compared to mammals, at the base of all teleost (Ts3R) and in a common ancestor of all salmonids 100-80 Mya (Ss4R) [15]. Of the Ts3R and Ss4R gene duplicates, $\sim 20$ and $55 \%$ respectively are still retained as expressed genes in the genome [16]. This dramatic increase in the number of homologous genes in salmon thus necessitates a careful annotation of PL synthesis and LP formation pathway genes and their tissue-specific expression regulation to improve our understanding of salmon PL metabolism.

In this paper we annotate and characterize gene regulation involved in PL synthesis and LP formation in different intestinal regions (SM, PC and $\mathrm{HG}$ ) during early developmental stages of salmon. Our aims are to (I) improve our understanding of the homologous genes, especially from Ss4R, and their contribution to salmonid specific features of regulation of PL metabolism pathways and to (II) specifically investigate the association of insufficient expression of PL and LP synthesis pathways in salmon fry with its higher dietary PL requirement.

\section{Methods}

\section{Fish, diet and sampling procedure}

Atlantic salmon eggs were hatched and cultivated at AquaGen Breeding Centre (Kyrksæterøra, Norway). From first-feeding, the fish were fed a normal commercial diet which satisfies the nutritional requirement of salmon, but without any additional PL supplement (Additional file 1). The diet was produced by EWOS AS (Bergen, Norway). Six salmon individuals were sampled at sizes of $0.16 \mathrm{~g}$ ( $n=6,1$ day before first feeding), $2.5 \mathrm{~g}$ ( $n=6,65$ days after first feeding) and $10 \mathrm{~g}(n=6$, 100 days after first feeding). The fish were euthanized by 1 g/L MS-222 (FINQUEL, Argent chemical labs, Washington, USA) buffered with same amount of sodium bicarbonate before dissection. The belly of the fish was dissected and immediately placed in $1 \mathrm{~mL}$ RNALater. Tissues were stored for $24 \mathrm{~h}$ at $4{ }^{\circ} \mathrm{C}$ for sufficient penetration of RNALater, before being dissected in sterile petri dish filled with RNALater. The entire intestinal tract was carefully separated from belly, connective tissues were removed and the intestinal contents were squeezed. Samples of stomach (SM, section between the end of esophagus and start of pyloric caeca), pyloric caeca (PC, section with fingerlike projections) and hindgut ( $\mathrm{HG}$, section after mid intestine) were dissected under dissecting microscope and immediately placed in in $2 \mathrm{~mL}$ cryo tubes 
(Thermo Fisher Scientific, Waltham, Massachusetts, USA) and transferred to $-80{ }^{\circ} \mathrm{C}$ before further analysis.

\section{RNA extraction, library preparation and transcriptome sequencing}

The RNA extraction and library preparation were carried out in Centre for Integrative Genetics (CIGENE, Ås, Norway). Tissues from two individuals $(n=2 \times 3)$ at 0 . $16 \mathrm{~g}$ were merged to obtain sufficient RNA for analysis. For individuals at 2.5 and $10 \mathrm{~g}$, the RNA was extracted individually $(n=1 \times 6)$. Total RNA was extracted from SM, PC and HG using RNeasy Plus Universal Kits (QIAGEN, Hilden, Germany), according to manufacturer's instruction. RNA concentration and purity were assessed by Nanodrop 8000 (Thermo Scientific, Wilmington, USA). RNA integrity was checked by Agilent 2100 Bioanalyzer (Agilent Technologies, Santa Clara, CA, USA). All samples had a RIN value $>8$, sufficient for transcriptome analysis. RNA libraries were prepared by using TruSeq Stranded mRNA Library Prep Kit (Illumina, San Diego, CA, USA), according to manufacturer's instruction. Samples were sequenced using $100 \mathrm{bp}$ single-end high-throughput mRNA sequencing (RNA-Seq) on Illumina Hiseq 2500 (Illumina, San Diego, CA, USA) in Norwegian Sequencing Centre (Oslo, Norway).

\section{Identification of genes and phylogenetic analysis}

The genes involved in PL synthesis and LP formation in Atlantic salmon (Salmo salar) were manually annotated by matching salmon to zebrafish (Danio rerio) orthologs from the KEGG reference pathway of glycerolphospholipid metabolism and other studies [17-19]. Ortholog group predictions were carried out using Orthofinder (v0.2.8) on proteins from eight fish species: zebrafish (Danio rerio), stickleback (Gasterosteus aculeatus), medaka (Oryzias latipes), Northern Pike (Esox lucius), grayling (Thymallus thymallus), rainbow trout (Oncorhynchus mykiss), coho salmon (Oncorhynchus kisutch), Atlantic salmon (Salmo salar), and two mammal outgroup species: human (Homo sapiens) and mouse (Mus musculus). The protein sequences within orthogroups were aligned to each other using MAFTT and maximum likelihood trees were estimated using FastTree. Orthogroup trees were subsequently split into smaller clan trees using an in-house $\mathrm{R}$ script (clanfinder.R, available from https:// github.com/srsand/Phylogenomics/blob/master/clanfinder.R). For zebrafish proteins in selected KEGG reference pathways, salmon proteins within the same protein clan tree were annotated using the zebrafish KEGG Orthology terms. The detailed information on Orthogroup prediction and phylogenetic analysis was published elsewhere [18]. All annotated salmon genes were grouped into a PL gene list and used for gene expression analysis (Additional file 2).

RNA-sequencing data and statistical analysis

Read sequences were quality trimmed, removing any Illumina TruSeq adapter sequence and low quality bases (Phred score $<20$ ) from read ends and length filtered (minimum length 40 bases) using cutadapt (v1.8.1), before being aligned to the salmon genome (ICSASG_v2) using STAR (v2.5.2a). Raw gene counts per sample were generated from read alignments using HTSeqcount (v0.6.1p1) and the NCBI salmon genome annotation (available for download at http://salmobase.org/ Downloads/Salmo_salar-annotation.gff3.gz). The uniquely mapped reads, aligned to exon regions, were counted for each gene in the annotation.

For each tissue type (SM, PC, HG), a differential expression analysis (DEA) was performed comparing all genes in $2.5 \mathrm{~g}$ and $10 \mathrm{~g}$ samples to the $0.16 \mathrm{~g}$ samples. Genes were filtered prior to DEA testing by a minimum count level of at least 1 count per million (CPM) in two or more samples, to remove genes with too few counts for testing. From raw counts, DEA was conducted using $R$ package edgeR (v3.8.6) with pairwise exact tests to produce gene fold changes and false discovery rate (FDR) adjusted $p$ values $(q)$. Genes with $q<0.05$ were considered to be differentially expressed genes (DEGs) between two test conditions. A KEGG ontology enrichment analysis (KOEA) was also conducted using edgeR. For KO terms, $p$ values were generated based on the number of DEGs compared to the total number of genes annotated to each $\mathrm{KO}$ term. The PL gene list was used to subset the total DEA results to find DEGs involved in the synthesis of PL and LP.

To visually compare expression levels between different genes and tissues, normalized counts in the form of transcripts per million (TPM) values were generated. Raw gene counts were first divided by their mRNA length in kilobases to normalize for transcript length, then divided by the total number of counts from each library to normalize for sequencing depth. Sample library sizes were normalized to each other using the edgeR TMM normalization method.

All RNA-Seq data analysis was preformed using R (v3. 2.4) and Bioconductor (v3.3). The pathway maps of PL and LP synthesis were produced using PathVisio. The heatmap was drawn using $\mathrm{R}$ package pheatmap. All other figures were produced using SigmaPlot for Windows Version 13.0.

\section{Results}

Annotation of PL pathway genes in salmon

To examine expression of genes involved in PL and LP synthesis in salmon, we created a list of all PL-related 
salmon genes in the pathways based on their zebrafish orthologs identified in previous studies. A total of 62 zebrafish genes involved in PL de-novo synthesis, lysoPL synthesis, PL turnover and LP synthesis pathways were used to identify PL metabolism genes in salmon. In total, 125 corresponding salmon homologs were identified based on their phylogenetic relationship to human and zebrafish orthologs. For each zebrafish gene, 1 or more salmon orthologs were identified. Due to the Ss4R WGD, $67 \%$ of the PL genes contain a salmon-specific duplicate in the genome. Homologous genes involved in the same enzymatic reaction were grouped into a family for comparison of gene expression. A summary of identification and nomenclature of $\mathrm{PL}$ genes is shown in Additional file 2.

\section{Tissue specific regulation of PL metabolism in the gut}

An average total of 22 million reads were sequenced from each library, out of which $\sim 85 \%$ were mapped to the salmon genome ICSASG_v2 (Additional file 3). From a total of 81,574 genes currently annotated in the salmon genome, 31,411 genes passed a minimum level of read counts for use in DEA. DEA was carried out on SM, PC, and HG separately to assess the extent of developmentally associated changes by comparing $2.5 \mathrm{~g}$ and $10 \mathrm{~g}$ salmon to $0.16 \mathrm{~g}$ (Additional file 4). The different intestinal regions differed greatly in the number of differentially expressed genes (DEGs, $q<0.05$ ), with $10 \%$ of genes differentially expressed in SM, and around $30 \%$ in PC and HG (Table 1). A KEGG ontology enrichment analysis (KOEA) on DEGs in SM, PC, and HG of salmon

Table 1 Number of significantly $(q<0.05)$ differentially expressed genes (DEGs) between 2.5, 10 and $0.16 \mathrm{~g}$ salmon

\begin{tabular}{lll}
\hline & DEGs & Percentage of total genes \\
\hline All genes & & \\
$2.5 \mathrm{~g}$ vs $0.16 \mathrm{~g} \mathrm{SM}$ & 3369 & $11 \%$ \\
$10 \mathrm{~g}$ vs $0.16 \mathrm{~g} \mathrm{SM}$ & 2897 & $9 \%$ \\
$2.5 \mathrm{~g}$ vs $0.16 \mathrm{~g} \mathrm{PC}$ & 8552 & $27 \%$ \\
$10 \mathrm{~g}$ vs $0.16 \mathrm{~g} \mathrm{PC}$ & 11,427 & $36 \%$ \\
$2.5 \mathrm{~g}$ vs $0.16 \mathrm{~g} \mathrm{HG}$ & 9052 & $29 \%$ \\
$10 \mathrm{~g}$ vs $0.16 \mathrm{~g} \mathrm{HG}$ & 10,475 & $33 \%$ \\
PL genes & & \\
$2.5 \mathrm{~g}$ vs $0.16 \mathrm{~g} \mathrm{SM}$ & 22 & $18 \%$ \\
$10 \mathrm{~g}$ vs $0.16 \mathrm{~g} \mathrm{SM}$ & 10 & $8 \%$ \\
$2.5 \mathrm{~g}$ vs $0.16 \mathrm{~g} \mathrm{PC}$ & 47 & $40 \%$ \\
$10 \mathrm{~g}$ vs $0.16 \mathrm{~g} \mathrm{PC}$ & 64 & $52 \%$ \\
$2.5 \mathrm{~g}$ vs $0.16 \mathrm{~g} \mathrm{HG}$ & 45 & $36 \%$ \\
$10 \mathrm{~g}$ vs $0.16 \mathrm{~g} \mathrm{HG}$ & 49 & $40 \%$ \\
\hline
\end{tabular}

The total number of genes was 31,411, with 124 genes involved in PL biosynthesis and LP formation pathways ( $P L$ genes) identified pathways which were significantly $(p<0.05)$ regulated between different stages of development (Additional file 5). This included the glycerophospholipid metabolism pathway, which was significantly regulated in PC and HG, but not SM.

The relative expression of genes involved in PL synthesis and LP formation showed categorization into three distinct tissue related clusters associated with developmental differences (Fig. 1). The genes in cluster 2 were characterized by having highest expression in PC, while the remaining genes were either highest expressed in SM (cluster 1) or HG (cluster 3). Only a few genes were down-regulated during the development of fish, whereas most genes showed onset of expression, especially in cluster 2 . The differentially expressed PL genes were annotated in Fig. 1. Similar to genome-wide changes in expression, PL genes PC and HG were much more responsive ( $40 \%$ DEGs) to development compared to SM (less than 20\% DEGs). Most DEGs were shared between PC and HG in cluster 2, while fewer DEGs were in other clusters. Moreover, the shared DEGs showed a much larger change in PC than in HG during development, resulting in increasing difference of expression between PC and HG as salmon grew.

\section{Regulatory divergence of homologous genes among developmental stages and intestinal sections among Ss4R duplicates}

The relative expression (TPM value) of all genes in the PL and LP synthesis pathways are summarized in Additional file 6. The salmon-specific (Ss4R) duplicate pairs of genes showed a significantly $(p<0.05)$ more similar expression than other duplicate pairs in each homologous gene family, regardless of developmental stages and intestinal sections (Fig. 2a). Moreover, the highest expression levels of the homologous genes were mostly found in PC rather than in SM or HG, which suggests that $\mathrm{PC}$ is the most important intestinal section for PL synthesis and LP formation in salmon (Additional file 6).

Pcyt1 family is a representative example of the regulatory complexity of homologous genes among intestinal sections and developmental stages (Fig. 2b). The pcyt1 family has 2 members in mammals (pcyt1a and pcyt1b), 4 members in zebrafish (pcyt1aa, pcyt1ab, pcyt1ba, and pcyt1bb) and 7 members in salmon (pcyt1aa, pcyt1ab_1, pcyt1ab_2, pcyt1ba_1, pcyt1ba_2, pcyt1bb_1, and pcyt1bb_2). Among all homologous genes, $p c y t 1 b b_{-} 1$ had much higher expression levels in PC than in SM and HG. It was also the most highly expressed homolog in all tissues. The expression level of pcyt1bb_1 in PC and HG both more than doubled as the fish grew from $0.16 \mathrm{~g}$ to $10 \mathrm{~g}$. The Ss4R homologous genes, pcyt1bb_1 and pcyt1bb_2, showed similar divergence in expression levels between tissues and developmental stages. The expressions of 


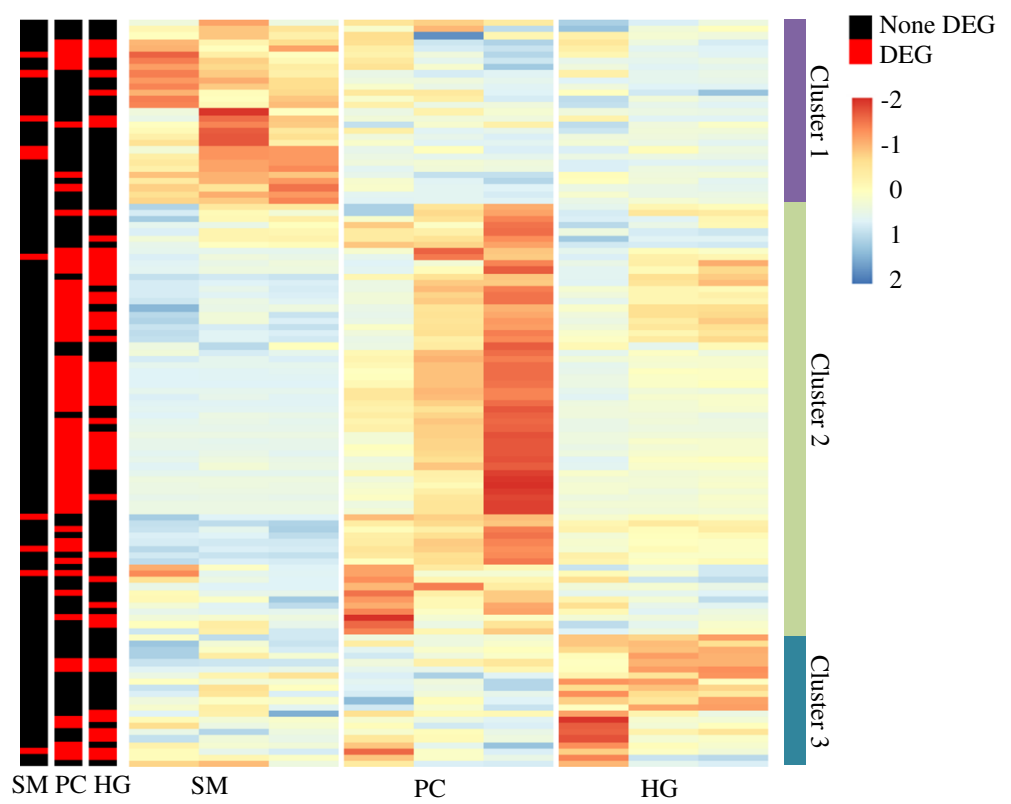

Fig. 1 Expression of genes in phospholipid and lipoprotein synthesis pathways between different intestinal regions of salmon. For each tissue the three columns represent $0.16 \mathrm{~g}, 2.5 \mathrm{~g}$ and $10 \mathrm{~g}$ samples from left to right. The color intensity is relative to the standard deviation from mean of TPM over developmental stages and tissues (row-scaled). Differential expressed genes (DEG, $q<0.05$ ) between $0.16 \mathrm{~g}, 2.5 \mathrm{~g}$ and $10 \mathrm{~g}$ samples were annotated in three columns, which represent stomach (SM), pyloric caeca (PC) and hindgut (HG) respectively from left to right

pcyt1ab_1 and pcyt1ba_1 genes were both higher in SM than PC and HG, while pcyt1aa, pcyt1ab_2, and pcyt1ba_ 2 were similarly expressed between the three tissues.

\section{Regulation of PL synthesis and LP formation pathways in PC}

It clear from our expression analyses (Fig. 1) that PC is the most transcriptionally active tissue, measured as expression levels of genes involved in PL and LP metabolic pathways. Therefore, PC was selected for a detailed study of the differences in expression of homologous genes between $0.16 \mathrm{~g}, 2.5 \mathrm{~g}$, and $10 \mathrm{~g}$ salmon (Fig. 3). The homologous genes in 18 families of key genes in PtdCho, PtdEtn and LP synthesis pathways were thus selected for in depth analyses.

In most gene families, one or two genes had much higher expression than their homologs, with all being up-regulated during development (Fig. 3). In the 11 families with two highly expressed genes, they are mostly salmon-specific duplicates from Ss4R WGD. In de-novo synthesis pathways, highly expressed genes were mostly significantly $(q<0.05)$ up-regulated from $0.16 \mathrm{~g}$ to $10 \mathrm{~g}$ (Fig. 3a). The highly expressed genes involved in the PtdCho synthesis pathway (chk, pcyt1, and chpt families) showed a much more pronounced increase compared to genes in other de-novo synthesis pathways. When we compared TPM of the highly expressed genes, lpin and plpp had slightly higher expression compared to $c d s$ family. Genes in ept family had much lower expression than other families in the PL de novo synthesis pathway. In lyso-PL synthesis pathways, the expression of lpcat $3 a$ was largely increased $(q<0.05)$ during development, whereas other lpcat genes remained stable $(q>0.05$, Fig. 3b). Lpgat1a and lpiat1 in other families in lysoPL pathways were also up-regulated $(q<0.05)$ during development (Additional file 7). All other genes in lyso-PL synthesis pathways were expressed at much lower levels than lpcat3a. Genes in LP formation pathway had much higher expression than in PL synthesis pathways (Fig. 3c). All highly expressed genes in LP formation pathway were largely up-regulated $(q<0.05)$ between 0.16 and $10 \mathrm{~g}$ salmon.

The proposed transcriptional regulation of PL synthesis and LP formation pathways is summarized in Fig. 4. By summarizing the change of the highly expressed genes in $\mathrm{PC}$, we found up-regulation of genes in the de-novo PtdCho and PtdEtn synthesis pathways and downregulation of genes in the de-novo PtdSer and PtdGro synthesis pathways between 0.16 and $10 \mathrm{~g}$ salmon (Fig. 4a). Other de-novo synthesis pathways were not changed during development. The Lyso-cardiolipin synthesis pathway was not changed, while other lyso-PL synthesis pathways were all up-regulated. Expression of genes in the phospholipid turnover pathways were not changed during development. The LP formation pathway was up-regulated between 0.16 and $10 \mathrm{~g}$ (Fig. 4b). 

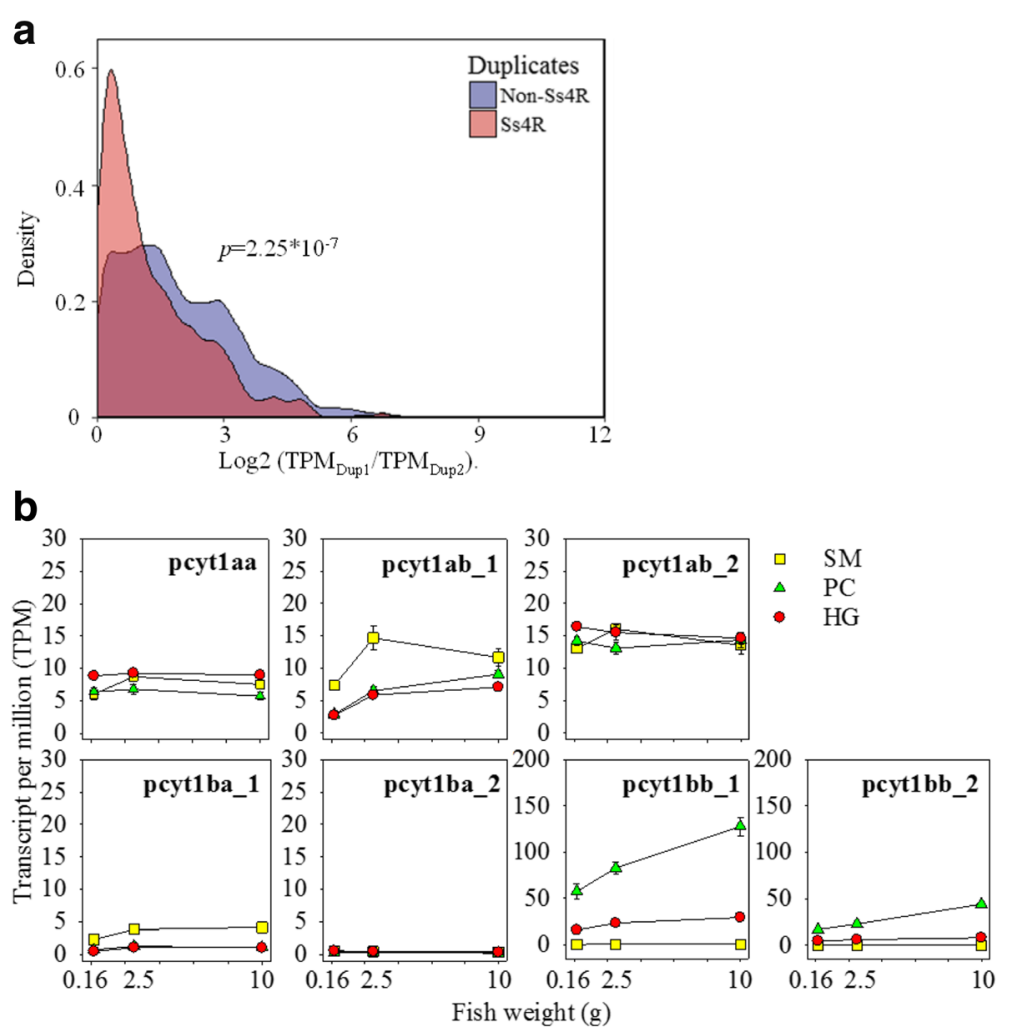

Fig. 2 Comparison of gene duplicates over developmental stages and intestinal regions. a The distribution of expressional differences between of genes in each homologous gene family. Gene expression in transcripts per million (TPM) was compared between pairs of every gene homolog, by taking the ratio of the higher expressed gene (TPM Dup1 $_{1}$ ) over the lower expressed (TPM Dup2). Expression ratios of gene pairs were calculated for each tissue and development stage. The density of expression ratios on a log2 scale is showed for Ss4R and non-Ss4R homolog gene pairs. A t-test between mean expression ratios of Ss4R and non-Ss4R showed a significant $\left(p=2.25 \times 10^{-7}\right)$ difference. $\mathbf{b}$ Comparison of pcyt1 genes in TPM over developmental stages and intestinal regions. The gene expressions were compared over three developmental stages $(0.16,2.5$ and $10 \mathrm{~g})$ in stomach (SM), pyloric caeca (PC) and hindgut (HG) of salmon. Numbers after underline indicates Ss4R gene duplicates specific in salmonids

\section{Discussion}

The objective of the present study was to explore the transcriptional changes of PL synthesis and LP formation pathways in different intestinal regions of salmon during early developmental stages. By integrating RNASeq data with a manually curated automated sequence ortholog prediction, we identified many DEGs in SM, PC, and HG during development of salmon. Most of those changes occurred between the onset of feeding at 0.16 and $2.5 \mathrm{~g}$, and with additional changes between 0.16 and $10 \mathrm{~g}$. This shows that the maturation of lipid metabolic pathways progressed for a substantial period following the completion of yolk sac reabsorption. By comparing the highly expressed genes in each family, we found a continuous increased capacity of PtdCho, PtdEtn, and LP synthesis in PC from 0.16 to $10 \mathrm{~g}$. This implies an increased capacity for PL and LP synthesis after onset of feeding.

Our results are in line with previous qPCR-based targeted gene studies in salmon in which several major PL biosynthetic genes were up-regulated in PtdCho and
PtdEtn synthesis between 2.5 and $10 \mathrm{~g}$ [20]. However, we did not observe a clear leveling-off of expression levels of synthesis genes at $10 \mathrm{~g}$, suggesting that the completion of intestinal maturation might take longer time to accomplish. This supports the hypotheses of a higher dietary PL requirement in salmon fry compared to larger fish [10].

The present study was the first to investigate expression differences between homologous genes in PL and LP synthesis pathways in salmon. Several homologous genes were identified that encode enzymes that catalyze the same reaction in PL and LP synthesis. In most cases, we found that one or two genes had much higher expression levels than their homologs for a given tissue, suggesting these genes to be key regulators in the pathways. However, the expression levels of the homologous genes appear to vary with both intestinal regions and developmental stages. This resulted in different genes dominating in expression levels in SM, PC and HG. The differences in expression are likely due to differences in function, subcellular localization, and developmental 


\section{a PL de-novo synthesis pathways}
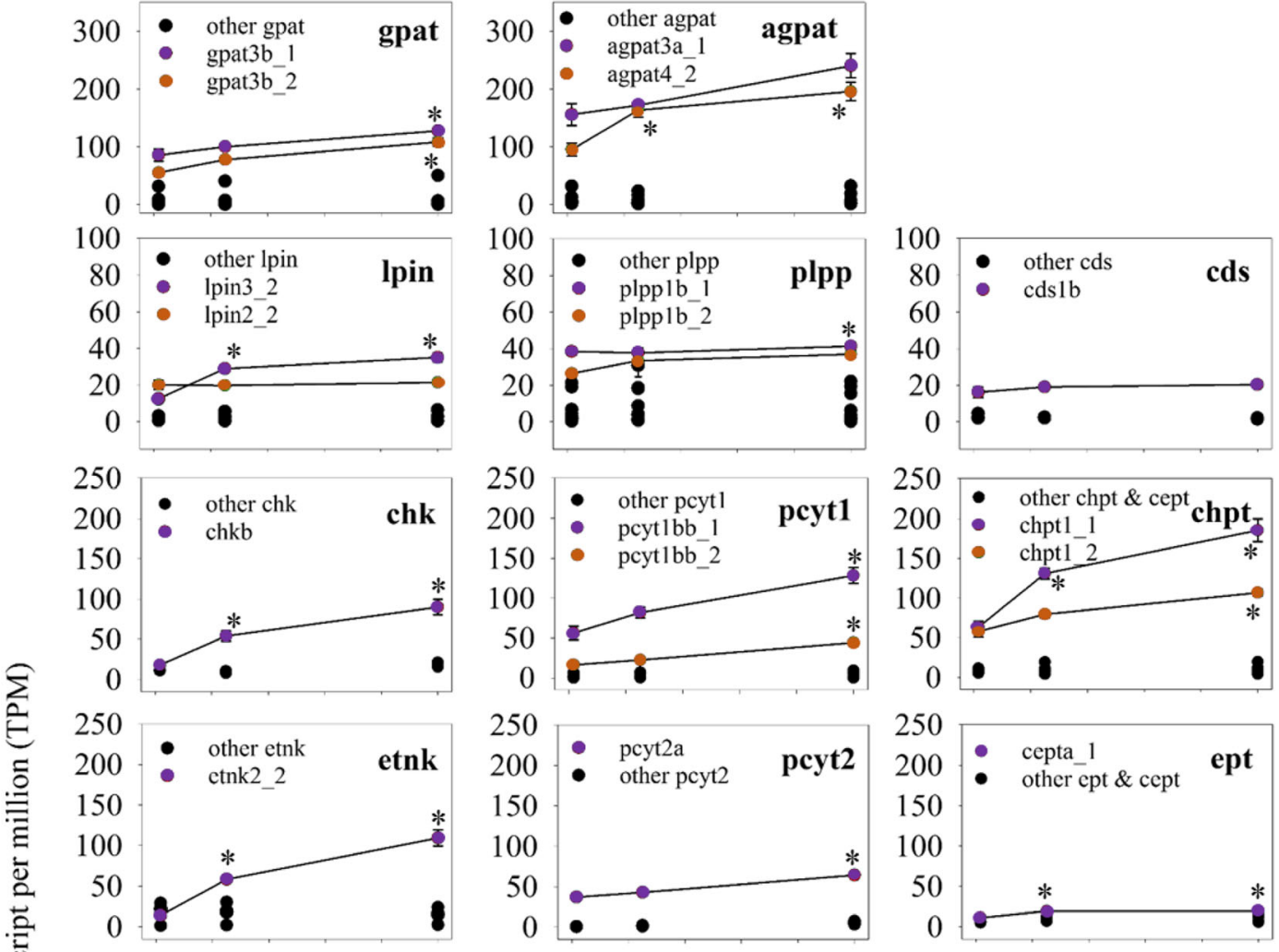

b Lyso-PL synthesis pathways

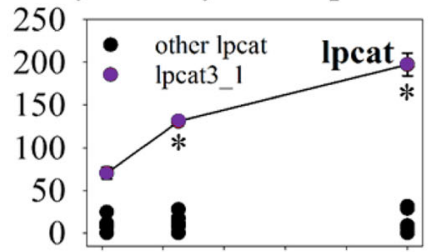

C Lipoprotein formation pathway
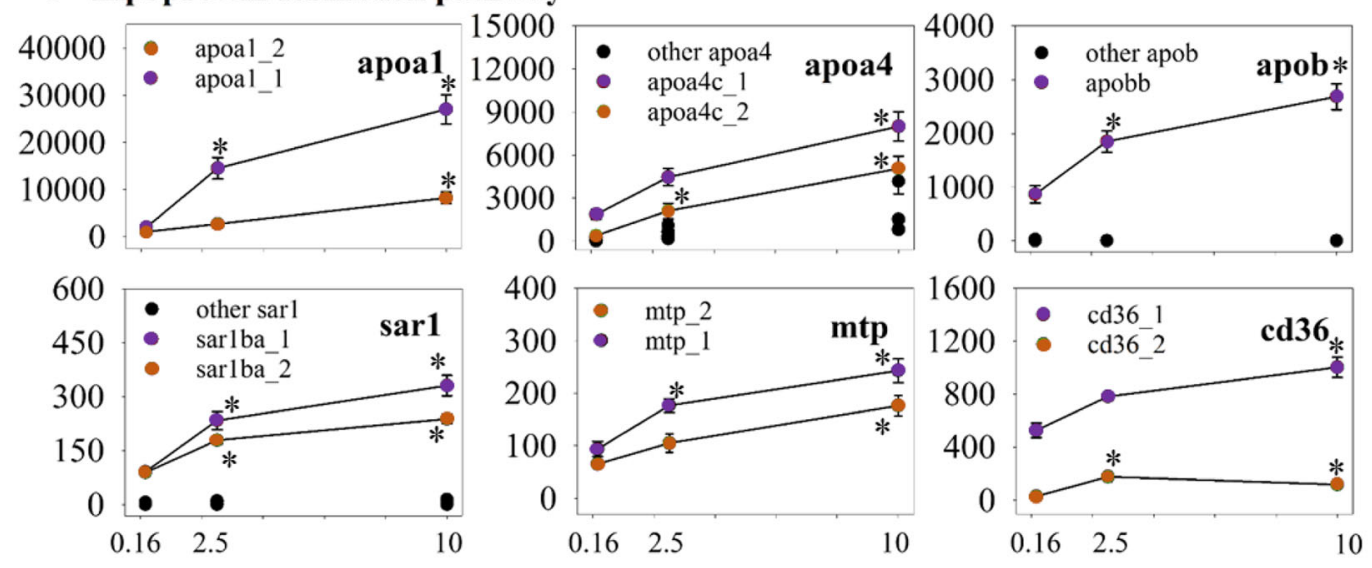

Fish weight $(\mathrm{g})$

Fig. 3 (See legend on next page.) 
(See figure on previous page.)

Fig. 3 Expressions of key genes in phospholipid and lipoprotein synthesis pathways in pyloric caeca at early stage of salmon. Eighteen families of homologous genes in phospholipid (PL) de-novo synthesis (a), lyso-phospholipid (lyso-PL) synthesis (b) and lipoprotein formation (c) pathways are shown for comparing their relative expression in transcripts per million (TPM) between 0.16, 2.5 and $10 \mathrm{~g}$ fish. Genes with high TPM are marked in purple and orange, while other genes were all marked in black. Points that significantly different in expression $(q<0.05)$ compared to $0.16 \mathrm{~g}$

a

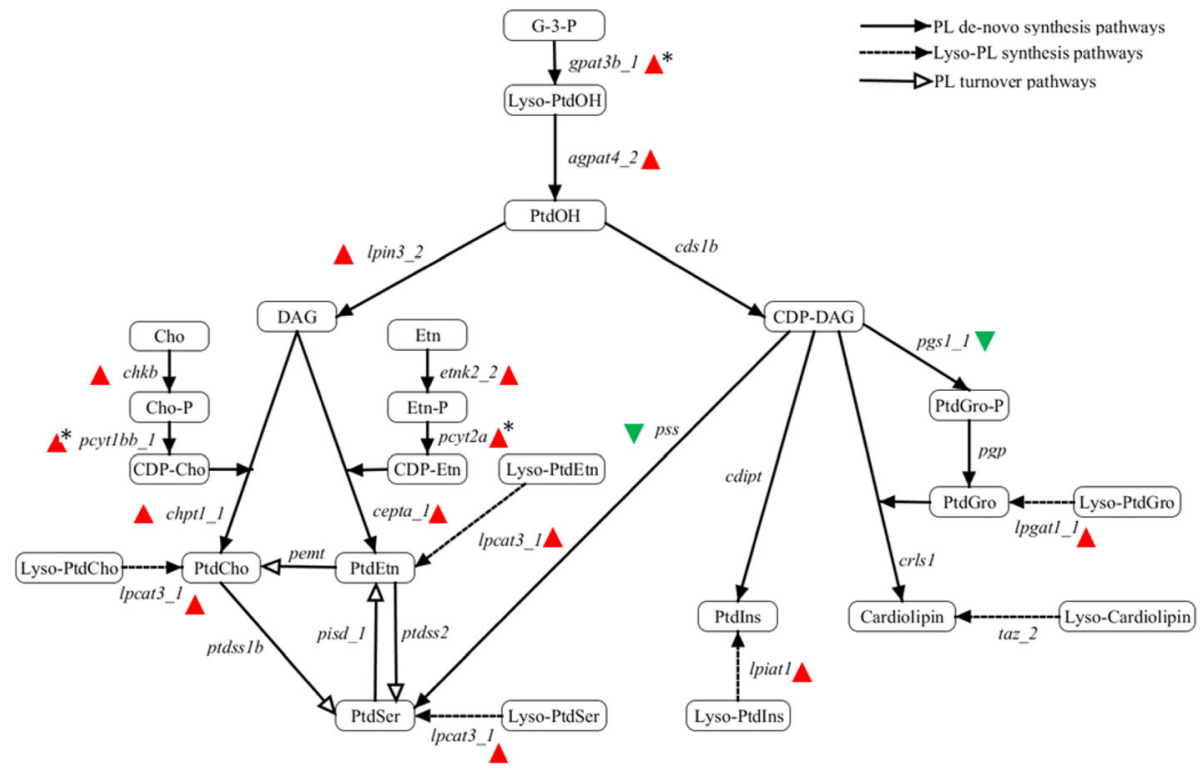

b

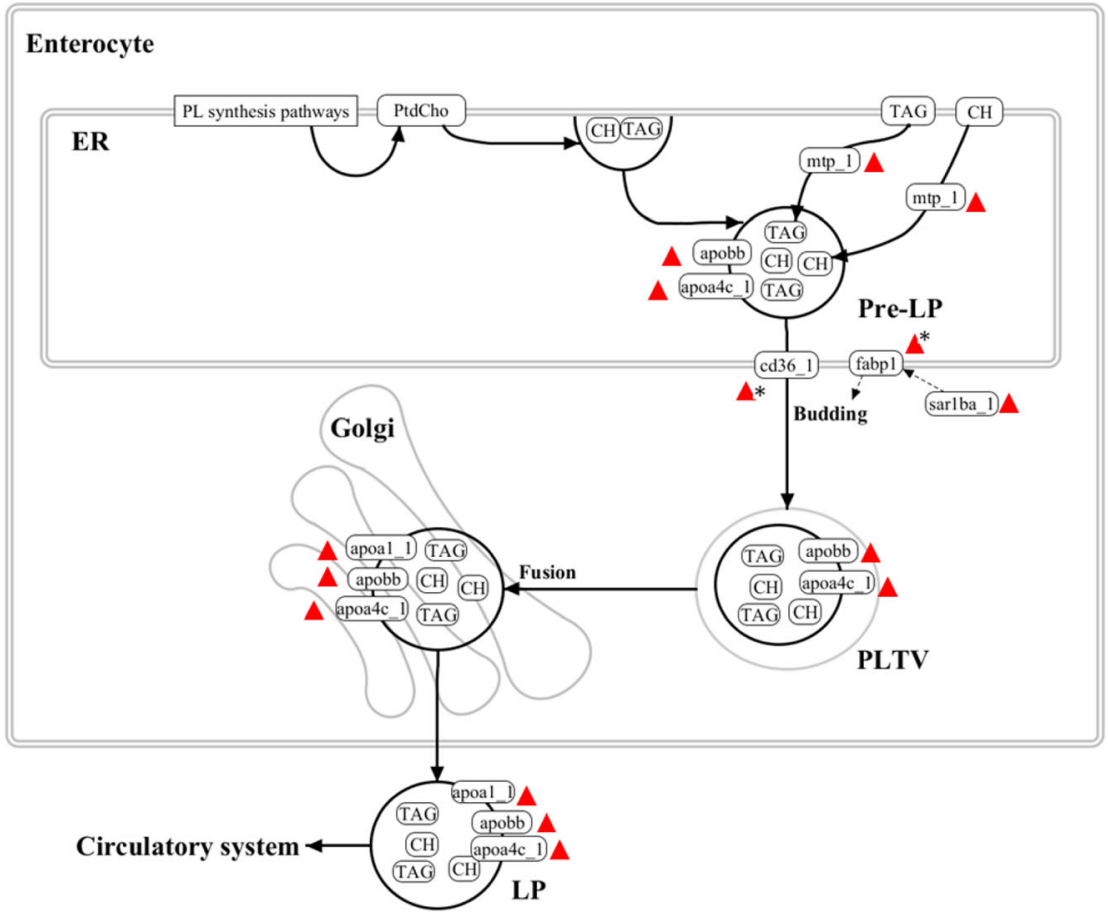

Fig. 4 (See legend on next page.) 
(See figure on previous page.)

Fig. 4 Comparison of phospholipid (PL) synthesis and lipoprotein (LP) formation pathways between 0.16, 2.5 and $10 \mathrm{~g}$ salmon. Colored triangles indicate the significantly $(q<0.05)$ up (red) or down (green) regulation of the highest expressed genes found in each enzymatic step of the pathways. Asterix indicates genes only significantly $(q<0.05)$ changed between $0.16 \mathrm{~g}$ and $10 \mathrm{~g}$. a PL de-novo synthesis, lyso-PL synthesis and PL turnover pathways in fish. Glycerol-3-phosphate (G-3-P) is first acylated by acyltransferases to phosphatidic acid (PtdOH), which can be transferred into diacylglycerol (DAG) or CDP-diacylglycerol (CDP-DAG) by phosphatidate phosphatase (plpp and lpin) or CDP-DAG synthetase (cds). DAG is utilized with CDP-choline (CDP-Cho) and CDP-ethanolamine (CDP-Etn) for synthesizing of phosphatidylcholine (PtdCho) and phosphatidylethanolamine (PtdEtn). CDP-DAG is utilized for synthesizing of phosphatidylserine (PtdSer), phosphatidylglycerol (PtdGro), phosphatidylinositol (Ptdlns) and Cardiolipin. b LP formation pathway in enterocyte of fish. PtdCho is synthesized on the membrane of endoplasmic reticulum (ER) through de-novo synthesis, turnover or lyso-PL pathway before used for pre-lipoprotein (Pre-LP) formation. Pre-LP is a nascent lipoprotein assembled by PtdCho, triacylglycerol (TAG), cholesterol (CH), apolipoprotein B (apob) and apolipoprotein AIV (apoa4). Pre-LP is then targeted to the Golgi apparatus via pre-lipoprotein transport vesicle (PLTV) generated by ER. The maturation of Pre-LP happens in Golgi, where apolipoprotein Al (apoa1) is added before secreting into circulatory system

stages of homologous genes in the tissues [1]. More interestingly, the salmon-specific homologous genes derived from Ss4R WGD were expressed at similar levels in different tissues and developmental stages, while other homologous genes seemed to have more differences in expression. This may suggest a functional divergence of Ss4R homologous genes was incomplete compared to homologs from other genome duplications. This supports the recent study in which $55 \%$ of the Ss4R homologous genes were found to have similar expression levels among 15 tissues in salmon [16].

The present study was the first to utilize RNA-Seq to investigate differences in global gene expression patterns among different intestinal regions in PL metabolism. PC is the predominant region for lipid absorption and transport in salmon [12, 13]. This is consistent with higher expression of genes involved in PtdCho, PtdEtn, and LP synthesis pathways in PC rather than SM and HG. As PtdCho is the predominant lipid class forming the membrane fraction of LP, the higher expression of genes in PtdCho synthesis pathway in PC suggests a high rate of LP production $[3,8]$. This has been confirmed by histological observation of large lipid droplets accumulating in midgut enterocytes of PL-deficient fish, while little droplets were found in fish fed dietary PtdCho [9, 10]. The expression of genes involved in PL synthesis in SM and HG were most likely related to other biological functions such as cell maintenance and metabolism. Almost no expression of LP formation genes was found in $\mathrm{SM}$, in agreement with the general observation that SM is not involved in lipid digestion, uptake, and transport. However, despite being expressed at low levels, many genes of the LP formation pathways were found in HG, suggesting some capacity to absorb and transport lipid in this intestinal region [14].

Several highly expressed genes in PL and LP synthesis pathways of salmon do not conform to the known function and regulation of these pathways in mammals. For example, it is believed that choline kinase (CK) $\alpha$ enzyme is critical for PtdCho maintenance in most tissues in mammals, whereas CKß enzyme is only essential in muscle tissue [21, 22]. In salmon, the expression of the chkb gene, encoding for CKß, was significantly elevated in PC after start feeding, whereas chka genes were unchanged. Similarly, the expressions of pcyt1a genes were relatively low in PC during early development, while pcyt1bb_1 notably increased in PC after onset of feeding. Therefore, we assume that the production of PtdCho for LP synthesis is probably through a compensatory pathway controlled by $c h k b$ and pcty $1 b$-encoded enzymes and activated in PC after switching to external feeding. On the other hand, there may be another pathway controlled by chka and pcyt1a-encoded enzymes, which produce PtdCho to maintain cell growth and survival. This suggestion agrees with previous studies pointing to the subcellular location of the enzymes [23, 24]. The CTP: phosphocholine cytidylyltransferase (CCT) $\alpha$, which is the product of the pcyt1a gene, is predominantly located in the nucleus. On the other hand, pcyt1b-encoded CCTß is localized in the endoplasmic reticulum (ER) and the cytosol, which could be utilized in synthesizing PtdCho for LP formation. However, as the level of gene expression does not always directly reflect relative importance of two similar enzymes in a pathway, the posttranslational modification like phosphorylation of CCT could also be critical in regulating the activity of enzymes without affecting the mRNA level [1].

\section{Conclusions}

The present study has provided new information on transcriptional events that may regulate PL synthesis and LP formation in salmon fry. By comparing the expression levels of homologous genes, we identified several genes which had highly expression among their homologs in PtdCho, PtdEtn, and LP synthetic pathways in PC of salmon. Those highly expressed genes were all up-regulated during development, suggesting the increasing capacity for PL synthesis and LP formation. Given the lower expression of PL genes in the early life of salmon and the link to PL requirement for growth, it 
seems likely that the critical supplementation point is during the onset of feeding, then decreases as the fish grows. The expression levels of the homologous genes in PL synthesis and LP formation pathways appeared to vary with both intestinal regions and developmental stages. This resulted in different transcripts dominating in abundance in SM, PC and HG. The salmon-specific homologous genes derived from Ss4R WGD were expressed similarly among different tissues and developmental stages, while homologous genes from other genome duplications seemed to have a different expression pattern. More studies on both gene expression and protein abundance are required to confirm these relationships during early stages of salmon development. Considering the present results on identification of key regulating genes in PL synthesis and lipid transport, we suggest a future study on the dietary requirement of PL at first-feeding stage, which focuses on the changes of key regulating genes involved in PL and LP synthesis pathways in PC of salmon.

\section{Additional files}

Additional file 1: Table S1. Composition and nutritional value of the diet used in current experiment. (DOCX $15 \mathrm{~kb}$ )

Additional file 2: Table S1. List of Atlantic salmon (Ssa) genes involved in phospholipid (PL) de-novo synthesis, lyso-PL synthesis, and lipoprotein $(L P)$ formation pathways. Nomenclature of salmon genes was based on their human ( $\mathrm{Hsa}$ ) and zebrafish (Dre) orthologs. Numbers after underline in Ssa names indicate salmon-specific gene duplicates. NCBI gene ID of salmon and zebrafish is also listed in table. Reference listed the origin of zebrafish genes used for identification of salmon genes. (DOCX 29 kb)

Additional file 3: Table S1. Summary of mapping statistics of all 45 samples used for RNA sequencing ( 2 fish each replicate $\times 3$ replicates $\times 3$ tissue in $0.16 \mathrm{~g}$ fish, 1 fish each replicate $\times 6$ replicates $\times 3$ tissue in 2.5 and $10 \mathrm{~g}$ fish). (DOCX $16 \mathrm{~kb}$ )

Additional file 4: Table S1. Result of differential expression ananlysis on all genes in stomach, pyloric caeca and hindgut of 2.5 and $10 \mathrm{~g}$ salmon both compared to $0.16 \mathrm{~g}$. (XLSX $14268 \mathrm{~kb}$ )

Additional file 5: Table S1. Result of KEGG ontology enrichment analysis on differential expressed genes (DEG) in stomach, pyloric caeca and hindgut of 0.16 and $2.5 \mathrm{~g}$ salmon both compared to $0.16 \mathrm{~g}$. (XLSX $65 \mathrm{~kb}$ )

Additional file 6: Table S1. Transcript per million (TPM) of all gene duplicates in phospholipid and lipoprotein synthesis pathways in stomach, pyloric caeca, and Hindgut of 0.16, 2.5 and $10 \mathrm{~g}$ salmon. (DOCX $32 \mathrm{~kb})$

Additional file 7: Table S1. Log2 fold change (LogFC) and adjusted $p$ value (q) of all gene duplicates in phospholipid and lipoprotein synthesis pathways in stomach, pyloric caeca and hindgut of 2.5 and $10 \mathrm{~g}$ salmon both compared to $0.16 \mathrm{~g}$. (DOCX $34 \mathrm{~kb}$ )

\section{Abbreviations}

agpat: 1-acylglycerol-3-phosphate acyltransferases; cd36: cluster of differentiation 36; cdipt: cdp-diacylglycerol-inositol 3-phosphatidyltransferase; CDP: Cytidine diphosphate; CDP-Cho: CDP-choline; CDP-DAG: CDP-diacylglycerol; CDP-Etn: CDPethanolamine; cds: CDP-DAG synthase; cept: CDP-choline/ethanolamine: diacylglycerol phosphotransferase; $\mathrm{CH}$ : Cholesterol; chk: choline kinase; ChoP: Phosphocholine; chpt: CDP-choline: diacylglycerol phosphotransferase; crls: Cardiolipin synthase; DAG: Diacylglycerol; ept: CDP-ethanolamine: diacylglycerol phosphotransferase; ER: Endoplasmic reticulum; etnk: ethanolamine kinase; Etn-P: Phosphoethanolamine; fabpl: Fatty acid binding protein, liver; G-3-
P: Glycerol-3-phosphate; gpat: glycerol-3-phosphate acyltransferase; Iclat: Iysocardiolipin acyltransferase; Ipcat: lysophosphatidylcholine acyltransferase; mboat: membrane bound o-acyltransferase; mtp: microsomal triglyceride transfer protein; pcyt1: choline-phosphate cytidylyltransferase; pcyt2: Ethanolaminephosphate cytidylyltransferase; pemt: phosphatidylethanolamine methyltransferase; pgp: phosphatidylglycero phosphatase; pgs: CDP-diacylglycerol: glycerol-3-phosphate phosphatidyltransferase; pisd: phosphatidylserine decarboxylase; plpp: phosphatidate phosphatase; pmt:: phosphoethanolamine nmethyltransferase; pss: CDP-diacylglycerol:serine phosphatidyltransferase; PtdCho: Phosphatidylcholine; PtdEtn: Phosphatidylethanolamine; PtdGro: Phosphatidylglycerol; Ptdlns: Phosphatidylinositol; PtdOH: Phosphatidic acid; PtdSer: Phosphatidylserine; ptdss: phosphatidylserine synthase; sar1: secretion associated, RAS related GTPase 1; TAG: Triacylglycerol; taz: tafazzin

\section{Acknowledgements}

Thanks to Dr. Maren Mommens and AquaGen AS for providing experimental facilities and other practical information. Thanks to Dr. Hanne Hellerud Hansen and Cigene for RNAseq analysis.

\section{Funding}

The study was supported by Department of Biology, Norwegian University of Science and Technology. We thank AquaGen AS for providing the fish and for contribution of running the experiment. The RNAseq analysis was financed by GenoSysFat NFR 244164. We also thank the China Scholarship Council for providing financial support to Yang Jin for his PhD study.

\section{Availability of data and materials}

The raw sequencing data are publicly available on European Nucleotide Archive under accession number PRJEB21981. The orthogroup prediction and phylogenetic analysis of salmon genes are available in Gillard et al. [18]. https://doi.org/10.1101/140442

\section{Authors' contributions}

YJ, RO, NS and YO comprehended the idea and designed the experiments. YJ sampled fish, prepared RNA-Seq and analyzed RNA-Seq data, and was a major contributor in writing the manuscript. M $\varnothing$ assisted in fish sampling. SK assisted in fish rearing and maintenance. GG and SS assisted in RNA-Seq data analysis. Annotation of salmon genes including orthogroup prediction and phylogenetic analysis were done by SS, AG, JV and JT. SS, RO and YO provided directions and critiques. All authors read and approved the final manuscript.

\section{Ethics approval}

The study was carried out within the Norwegian animal welfare act guidelines, in accordance with EU regulation (EC Directive 86/609/EEC) and approved by the Norwegian Animal Research Authority (NARA).

\section{Competing interests}

The authors declare that they have no competing interests.

\section{Publisher's Note}

Springer Nature remains neutral with regard to jurisdictional claims in published maps and institutional affiliations.

\section{Author details}

${ }^{1}$ Department of Biology, Faculty of Natural Sciences, Norwegian University of Science and Technology, NO-7491 Trondheim, Norway. ${ }^{2}$ Centre for Integrative Genetics, Department of Animal and Aquacultural Sciences, Norwegian University of Life Sciences, Ås, Norway. ${ }^{3}$ Department of Chemistry, Biotechnology and Food Science, Norwegian University of Life Sciences, Ås, Norway. ${ }^{4}$ AquaGen AS, Postboks 1240, Sluppen, N-7462 Trondheim, Norway.

Received: 6 July 2017 Accepted: 8 April 2018 Published online: 16 April 2018

\section{References}

1. Vance JE, Vance DE. Phospholipid biosynthesis in mammalian cells. Biochem Cell Biol. 2004;82(1):113-28.

2. Luchoomun J, Hussain MM. Assembly and secretion of chylomicrons by differentiated Caco-2 cells: nascent triglycerides and preformed phospholipids 
are preferentially used for lipoprotein assembly. J Biol Chem. 1999;274(28): 19565-72.

3. Zilversmit DB. The composition and structure of lymph chylomicrons in dog, rat, and man. J Clin Invest. 1965;44(10):1610-22.

4. Poston HA. Effect of body size on growth, survival, and chemicalcomposition of Atlantic Salmon fed soy lecithin and choline. Prog Fish Cult. 1990;52(4):226-30

5. Kjørsvik E, Olsen C, Wold PA, Hoehne-Reitan K, Cahu CL, Rainuzzo J, Olsen Al, Øie G, Olsen Y. Comparison of dietary phospholipids and neutral lipids on skeletal development and fatty acid composition in Atlantic cod (Gadus morhua). Aquaculture. 2009;294(3-4):246-55.

6. Dapra F, Geurden I, Corraze G, Bazin D, Zambonino-Infante JL, FontagneDicharry S. Physiological and molecular responses to dietary phospholipids vary between fry and early juvenile stages of rainbow trout (Oncorhynchus mykiss). Aquaculture. 2011;319(3-4):377-84.

7. Sargent JR, Henderson RJ, Tocher DR. The lipids. In: Halver JE, editor. Fish nutrition, vol. 2. New York: Academic Press; 1989.

8. Tocher DR, Bendiksen EÅ, Campbell PJ, Bell JG. The role of phospholipids in nutrition and metabolism of teleost fish. Aquaculture. 2008;280(1-4):21-34.

9. Taylor JF, Martinez-Rubio L, del Pozo J, Walton JM, Tinch AE, Migaud H, Tocher DR. Influence of dietary phospholipid on early development and performance of Atlantic salmon (Salmo salar). Aquaculture. 2015:448:262-72.

10. Olsen RE, Dragnes BT, Myklebust R, Ringo E. Effect of soybean oil and soybean lecithin on intestinal lipid composition and lipid droplet accumulation of rainbow trout, Oncorhynchus mykiss Walbaum. Fish Physiol Biochem. 2003;29(3):181-92.

11. De Santis C, Taylor JF, Martinez-Rubio L, Boltana S, Tocher DR. Influence of development and dietary phospholipid content and composition on intestinal transcriptome of Atlantic Salmon (Salmo salar). PLoS One. 2015; 10(10):e0140964

12. Denstadli V, Vegusdal A, Krogdahl A, Bakke-McKellep AM, Berge GM, Holm $\mathrm{H}$, Hillestad M, Ruyter B. Lipid absorption in different segments of the gastrointestinal tract of Atlantic salmon (Salmo salar L.). Aquaculture. 2004; 240(1-4):385-98.

13. Røsjø C, Nordrum S, Olli JJ, Krogdahl A, Ruyter B, Holm H. Lipid digestibility and metabolism in Atlantic salmon (Salmo salar) fed medium-chain triglycerides. Aquaculture. 2000;190(1-2):65-76

14. Calzada A, Medina A, Canales MLG. Fine structure of the intestine development in cultured sea bream larvae. J Fish Biol. 1998;53(2):340-65

15. Macqueen DJ, Johnston IA. A well-constrained estimate for the timing of the salmonid whole genome duplication reveals major decoupling from species diversification. Proc Biol Sci. 2014;281(1778):20132881.

16. Lien S, Koop BF, Sandve SR, Miller JR, Kent MP, Nome T, Hvidsten TR, Leong $J S$, Minkley DR, Zimin A, et al. The Atlantic salmon genome provides insights into rediploidization. Nature. 2016;533(7602):200-5.

17. Lykidis A. Comparative genomics and evolution of eukaryotic phospholipid biosynthesis. Prog Lipid Res. 2007:46(3-4):171-99.

18. Gillard G, Harvey TN, Gjuvsland A, Jin Y, Thomassen M, Lien S, Leaver M, Torgersen JS, Hvidsten TR, Vik JO, et al. Diet and life stage associated remodeling of lipid metabolism regulation in the duplicated Atlantic salmon genome. bioRxiv. 2017;

19. Siddiqi S, Mansbach CM. Dietary and biliary phosphatidylcholine activates PKC zeta in rat intestine. J Lipid Res. 2015;56(4):859-70.

20. Carmona-Antonanzas G, Taylor JF, Martinez-Rubio L, Tocher DR. Molecular mechanism of dietary phospholipid requirement of Atlantic salmon, Salmo solar, fry. Biochim Biophys Acta Mol Cell Biol Lip. 2015;1851(11):1428-41.

21. Gallego-Ortega D, Ramirez de Molina A, Ramos MA, Valdes-Mora F, Barderas MG, Sarmentero-Estrada J, Lacal JC. Differential role of human choline kinase alpha and beta enzymes in lipid metabolism: implications in cancer onset and treatment. PLoS One. 2009;4(11):e7819.

22. Sher RB, Aoyama C, Huebsch KA, Ji S, Kerner J, Yang Y, Frankel WN, Hoppel $C L$, Wood PA, Vance DE, et al. A rostrocaudal muscular dystrophy caused by a defect in choline kinase beta, the first enzyme in phosphatidylcholine biosynthesis. J Biol Chem. 2006;281 (8):4938-48.

23. Lykidis A, Baburina I, Jackowski S. Distribution of CTP : phosphocholine cytidylyltransferase (CCT) isoforms - identification of a new CCT beta splice variant. J Biol Chem. 1999;274(38):26992-7001.

24. Wang YL, Macdonald JIS, Kent C. Identification of the nuclear-localization signal of rat-liver Ctp-Phosphocholine Cytidylyltransferase. J Biol Chem. 1995;270(1):354-60.

\section{Ready to submit your research? Choose BMC and benefit from:}

- fast, convenient online submission

- thorough peer review by experienced researchers in your field

- rapid publication on acceptance

- support for research data, including large and complex data types

- gold Open Access which fosters wider collaboration and increased citations

- maximum visibility for your research: over $100 \mathrm{M}$ website views per year

At BMC, research is always in progress.

Learn more biomedcentral.com/submissions 